\title{
HOW DOES THYROIDECTOMY FOR BENIGN THYROID DISEASE IMPACT UPON QUALITY OF LIFE? A PROSPECTIVE STUDY.
}

\section{Carolyn R Chew MBBS MPHTM ${ }^{1}$, Synn Lynn Chin FRACS ${ }^{1}$, Tracey Lam FRACS $^{1}$, Allison Drosdowsky BA BSc MPH ${ }^{2,3}$, Steven TF Chan PHD FRACS ${ }^{4}$, Laura Chin-Lenn MBBS MEpi FRACS ${ }^{1}$}

1. Endocrine Surgery, Department of Surgery, Western Hospital, Melbourne, Victoria, Australia

2. Department of Cancer Experiences Research, Peter MacCallum Cancer Centre, Victoria, Australia

3. Sir Peter MacCallum Department of Oncology, University of Melbourne, Victoria, Australia

4. Department of Surgery, North West Academic Centre, The University of Melbourne, Victoria, Australia

\section{Author Contact Information}

1. Carolyn R Chew

Email: Carolyn.chew@wh.org.au

2. Synn Lynn Chin

Email: synnlynn@gmail.com

3. Tracey Lam

Email: Tracey.Lam@wh.org.au

4. Allison Drosdowsky

Email: Allison.drosdowsky@petermac.org

5. Steven TF Chan

Email: stfc@unimelb.edu.au

6. Laura Chin-Lenn (Corresponding Author)

Email: laura.chin-lenn@mh.org.au

Correspondence to: Western Health, 40 Gordon St Footscray Victoria Australia 3011

Author Contributions: All authors discussed the results and contributed to the final manuscript. LC and TL conceived the original idea, study design and ethics application. TL, SC and CC recruited patients, collected surveys and collated data from patient medical records. LC, TL, SC, STFC, CC and AD contributed to the interpretation of the results. AD and STFC performed the statistical analysis. CC and SC took the lead in writing the manuscript with support from LC, STFC, TL and AD. All authors provided critical feedback and helped shape the research, analysis and manuscript. LC supervised the project.

This is the author manuscript accepted for publication and has undergone full peer review but has not been through the copyediting, typesetting, pagination and proofreading process, which may lead to differences between this version and the Version of Record. Please cite this article as doi: $10.1111 /$ ans.16342

This article is protected by copyright. All rights reserved. 


\section{Running title: Thyroid Quality of Life Benign Disease}

Abstract Word Count: 248

Manuscript word count: 2791

Tables: 6

Preliminary data presented at RACS ASC meeting, Brisbane 2016.

Abstract

\section{Background}

Choosing which patients to recommend surgery for benign thyroid conditions can be difficult due to the subjective nature of compressive thyroid and hormonal symptoms. The aim of this prospective study was to analyse changes in Quality of Life (QOL) following thyroid surgery using a validated disease- specific assessment tool, the Thyroid Patient Reported Outcome (ThyPRO) questionnaire. Methods

Participants undergoing elective thyroid surgery for benign conditions were recruited. Patient demographics and clinical data were collected. ThyPRO consists of 85 questions grouped into 13 physical, mental and social symptom domains. Patients completed a ThyPRO questionnaire preoperatively and at six weeks and six months postoperatively. ThyPRO items were scored according to protocol to produce thirteen subscales. Repeated measures linear models with no random effects were performed using data for each outcome.

Results

Results were available for a total of 72 patients. The sample was predominately female $(n=63,88 \%)$ with average age 49.8 years. The majority of patients underwent surgery for multi-nodular goitre. At six weeks post-operatively, 
significant improvement was demonstrated in the goitre, hypothyroid, hyperthyroid and anxiety symptom domains. At six months post operatively, significant improvement was demonstrated in all but four domains. No domains demonstrated significant increase in impairment post-operatively.

Conclusions

Patients had significant improvement in nine of thirteen symptom domains following surgery. Patients did not experience a negative impact on QOL following surgery. Further studies with larger patient cohorts may be able to identify potential preoperative predictive factors for a post- operative improvement in QOL for benign thyroid disease.

Keywords: Thyroid, THYPRO, Benign, Quality of Life, thyroidectomy 


\section{Introduction}

Thyroid disease affects around $10-15 \%$ of the adult population with a female predominance. ${ }^{1}$ Most are benign conditions and encompass a heterogeneous group of clinical disease entities, sometimes with a combination of anatomical compressive symptoms and hormonal dysfunction. The reasons for seeking medical attention are often non-specific and difficult to measure, particularly "pressure symptoms" (e.g. choking sensation, difficulty breathing, dysphagia) and mood disturbance. Preoperatively, it is not always possible to demonstrate a pathophysiological relationship between these symptoms and the underlying disease. Patients' perceived changes in quality of life following thyroidectomy are significant, and include persistence, worsening or emergence of symptoms even in the absence of a complication such as recurrent laryngeal nerve injury or hypoparathyroidism (transient or permanent). ${ }^{2}$

The impact of thyroidectomy for benign thyroid disease on quality of life (QOL) has been infrequently studied. Non-disease specific health-related QOL surveys (e.g. SF-36), ${ }^{3}$ symptom specific QOL surveys (e.g. SWAL-QOL), ${ }^{4}$ or custom-made assessment scales ${ }^{5}$ have shown conflicting results with some describing no improvement in QOL and ongoing voice and swallowing problems, ${ }^{2}$ while some describing improvement. ${ }^{6}$ The Thyroid Patient Reported Outcome (ThyPRO) questionnaire is a validated disease- specific assessment tool. To date, six studies have used ThyPRO to assess QOL following thyroidectomy for benign disease. ${ }^{7-12}$ Several of these studies evaluating the impact of thyroidectomy on QOL for benign thyroid disease have demonstrated post-operative improvement in all domains. ${ }^{8-9,11}$ 
Given surgery for benign disease does not confer a survival benefit, treatment should not further impair QOL and should actually be expected to improve QOL in the post-operative period. Thus, patient reported, disease specific QOL outcomes are important to inform clinicians and patients regarding treatment expectations.

If the effect of surgery on quality of life (QOL) can be predicted, it would enable a more informed discussion with patients related to the risks and benefits of surgery. The objectives of this study are to investigate the impact of thyroidectomy for benign thyroid disease on QOL in an Australian population and to evaluate whether there are pre-operative factors that may predict patients who will achieve symptom benefit from surgery.

\section{Materials and Methods}

A prospective longitudinal observation study was conducted by the Endocrine Surgical Unit at Western Health, Melbourne, Australia. All patients were assessed and consented for surgery by consultant surgeons on the Endocrine Surgical Unit. Patients over the age of 18 undergoing total or hemi-thyroidectomy for benign thyroid disease between from July 2014 to January 2016 were invited to participate. Patients were recruited at their surgical or pre-admission outpatient appointments. Patients were excluded if they were pregnant, non-English speaking, or had suspected malignancy pre-operatively or confirmed on post-operative histopathology. Patient demographics, thyroid function, pre-operative imaging, histopathological diagnosis, body mass index (BMI) and post-operative morbidity were prospectively collected. 
Thyroidectomy was performed by uniform, capsular dissection, with routine intraoperative nerve monitoring under the care of specialist endocrine surgeons. Recurrent laryngeal nerve palsy was detected based on clinical assessment, with symptomatic patients undergoing flexible nasoendoscopy to evaluate vocal cord movement. Hypocalcaemia was defined as a corrected serum calcium of less than $2.10 \mathrm{mmolL}^{-1}$. Hypocalcaemia and recurrent laryngeal nerve palsy were defined as transient if they resolved within six months of operation and persistent if they extended beyond six months.

Thyroid patient-reported outcome (ThyPRO) was chosen for patient assessment, as it is as a validated and standardized disease-specific quality of life questionnaire..$^{7,13-15}$ It comprises 84 items assessing physical, mental and social domains of health. Each item is rated on a five-point scale, from zero (least symptomatic) to four (most symptomatic). Items are summarized into 13 domains (including goitre symptoms, hyper- or hypothyroid symptoms, emotional well-being and cosmetic concerns) and one score for overall QOL. Each domain generates a score from 0-100, with higher scores indicating decreasing QOL and lower scores indicating better QOL. ThyPRO demonstrates good internal reliability, test-retest reliability (intra-class correlations ranging from 0.77-0.89) and known groups validity. ${ }^{13,15-18}$ ThyPRO is the recommended health-related quality of life assessment for patients with benign thyroid diseases. ${ }^{19}$

Patients were allocated an anonymous study number and data entered into an anonymised spreadsheet. ThyPRO was administered on paper and collected at 
three time points: pre-operatively, at the six-week post-operative appointment and six months post-operatively. Informed written consent was obtained at the time of the initial ThyPRO questionnaire. The final six-month questionnaires were collected by mail. Non-responders were followed up with up to two telephone calls. Patients who did not complete all three questionnaires were excluded from final analysis. The study was approved by the Western Health Ethics committee.

\section{Statistical analysis}

Descriptive statistics were generated for participants' demographic, clinical and thyroid outcome measures. The ThyPRO items were scored according to protocol to produce thirteen subscales. ${ }^{13}$ Changes over time in each subscale of the ThyPRO tool were assessed using repeated measures models. All available data was included in each model, and comparisons were performed for each pair of time points (pre-surgery, six weeks post-surgery and six months post-surgery). To assess whether any demographic and clinical characteristics were associated with change in scores between pre-surgery and six months post-surgery, univariate linear regression was performed using a pre-specified set of explanatory variables. All analyses were performed in $\mathrm{R}^{20}$ and the nlme package was used for the mixed models. ${ }^{21}$

\section{Results}

Patient Population

A total of 99 patients were recruited to the study and completed the preoperative questionnaire. Twenty-seven patients were excluded: four had malignancy 
on histological examination and 23 were lost to follow up. The study group who completed three questionnaires ultimately consisted of 72 patients. Patient sociodemographic characteristics are summarized in Table 1. Sample clinical characteristics are outlined in Table 2 . The majority of patients were euthyroid, and almost half underwent surgery for multinodular goitres.

Surgical complications included one patient who had temporary recurrent laryngeal nerve palsy (1.4\%). No patients had a permanent clinically identified recurrent laryngeal nerve injury. Eighteen patients had temporary hypoparathyroidism, with one patient experiencing permanent hypoparathyroidism (1.4\%).

\section{Patients lost to follow up}

Twenty-three patients with benign disease were lost to follow up following completion of the baseline questionnaire. Socio-demographic and clinical characteristics are outlined in Table S1. The lost to follow up group were younger (42.6 vs 49.8 years old) and more likely to be smokers, but there was no other significant difference compared with the study group. Of those who did not complete post-operative surveys, all were reviewed at routine post-operative appointments. Transient complications included recurrent laryngeal nerve palsy $(n=1)$, symptomatic seroma $(n=1)$ and hypoparathyroidism $(n=3)$. No patients had permanent complications (i.e. permanent recurrent laryngeal nerve injury or permanent hypoparathyroidism). 
There were no significant differences $(p<0.05)$ between the baseline ThyPRO scores in the study group and the group lost to follow up, using an independent samples ttest. (Table S2).

\section{Thyroid Outcomes}

Sub-scale scores for the ThyPRO are summarized in Table S3. Change in estimates between pre-operative vs. six weeks post-operatively and six months postoperatively as well as post- operative changes at six weeks vs. six months are shown in Table 3. None of the domains demonstrated significant worsening at postoperative six week or six-month review.

Thyroid hormone dysfunction (Hyper- and hypothyroidism, tiredness)

The majority of patients our patients were euthyroid $(n=51,69.9 \%)$. Twenty $(27.4 \%)$ had biochemical evidence of hyperthyroidism prior to surgery and all of these patients reported hyperthyroid symptoms. Only one patient $(1.4 \%)$ had biochemical hypothyroidism. Thyroid dysfunction scores (hyperthyroid and hypothyroid domains) showed significant improvement between baseline and 6-week post-operative assessment.

Of all domains, tiredness was the most impaired score (baseline score of 57.1). This improved six weeks following surgery, but there was no further improvement at six months.

All three domains (hypothyroid symptoms, hyperthyroid symptoms and tiredness) showed statistically significant improvement at the six-month postoperative review compared with pre-operatively. 


\section{Goitre Symptoms}

Half of our study cohort had thyroid surgery due to goitre related symptoms $(n=36,49.3 \%)$. Goitre domain scores showed significant improvement at six weeks following surgery with continuing improvement at six months. $(p<0.005$, Table 3$)$. Eye and Cosmetic Symptoms

Eye symptoms improved significantly between baseline and six weeks postoperative scores $(p<0.01)$, and six-month post-operative scores $(p<0.05)$. Cosmetic scores did not change.

Mental and Social Domains

Baseline anxiety domain scores showed statistically significant improvement at six weeks postoperatively $(p<0.005)$, which remained significant at six months $(p<0.005)$. Cognition and depressivity domain scores demonstrated significant improvement between baseline and six months $(p<0.05)$. Emotional susceptibility scores were impaired at baseline but did not show significant improvement postoperatively $(p=0.052)$.

Social impairment was the least affected domain at baseline and six months post-operatively. There were no significant changes in domain scores for social life and sex life. Daily life impairment showed significant improvement at six weeks $(p=0.022)$ and six months $(p<0.01)$.

Patients also gave an overall score for quality of life at pre-operative, six-week post-operative and six-month post-operative reviews. At six-month post operatively, almost $60 \%$ of patients $(n=43)$ reported that their thyroid disease did not have a 
negative effect on their quality of life, compared with only $32 \%$ of patients preoperatively $(n=23)$.

Further univariate regression analysis was performed for possible demographic and clinical characteristics that may have predicted change in domain scores. Demographic characteristics analysed were age, gender, body mass index (BMI), smoking status and American Society of Anaesthesiologists (ASA) score. ${ }^{22}$ Clinical characteristics included TSH level, indication and type of surgery, complications, final histology and specimen weight. None of these variables were significantly associated with a higher improvement in QOL.

\section{Discussion}

Benign thyroid disease represents a heterogeneous population with varying levels of hormonal dysfunction. Symptoms, which may be due to a combination of anatomical or hormonal alterations, are non-specific and may not correlate with the underlying pathology. As surgery for benign thyroid disease does not confer survival advantage, benefits, namely symptomatic relief and improvement in QOL must outweigh the risk of post-operative complications. There are only a handful of published studies which evaluate QOL following benign thyroid surgery. ${ }^{7-12}$ This is the first study in Australia using ThyPRO to assess the impact of surgery on quality of life amongst patients with benign thyroid disease. At six months post-operatively, only four domains (social impairment, sex life, cosmesis, emotional susceptibility) had scores that were unchanged, with the remaining nine out of thirteen domains 
showing statistically significant improvement. Interestingly, a number of these domains were not significantly improved at six weeks post-operatively with the improvement only becoming apparent at six-month review. We would hypothesise that this may be after the patient has recovered fully from surgery and their temporary morbidity such as transient hypoparathyroidism or transient nerve paresis.

In keeping with similar ThyPRO studies looking at thyroidectomy for benign disease, tiredness was the most impaired pre-operative domain and showed statistically significant improvement post-operatively. ${ }^{7-12}$ In our study, tiredness was the also most impaired score, with a baseline score of 57.1. As point of reference, tiredness was also the highest scoring domain amongst a normal Danish population sample with a score of $35 .^{7}$ Thus, whilst an important symptom of thyroid disease, tiredness is not specific to thyroid disease and may be attributed to other causes that are either pre-existing or undiagnosed.

Goitre symptoms continued to show significant improvement over time. This finding is in agreement with other studies, where goitre symptoms all showed significant improvement following surgery for benign thyroid disease. ${ }^{7-12}$ Goitre related symptoms, namely dysphagia, dyspnoea, voice change and neck discomfort have previously been reported in separate QOL studies. These symptoms are included within the goitre domain of ThyPRO, however some, such as dysphagia and voice changes, may indirectly influence other domains, particularly social life. Thyroid surgery has been shown to lead to significant improvements in QOL using a generic health QOL questionnaire (SF-36) ${ }^{3}$ and validated swallowing 
questionnaires. ${ }^{4,6}$ Impairment of voice-related QOL is seen in high frequency amongst patients with benign thyroid disease and can lead to negative impacts on social functioning. ${ }^{23}$ Furthermore, voice alterations and dysphagia are commonly seen in post-operative populations even in the absence of RLN palsy. Despite this, objective measures such as speech pathology assessment and ultrasound examination of laryngeal mobility did not correlate to patient-reported symptoms, ${ }^{23,24}$ highlighting the clinical importance of assessing patient-reported QOL outcomes.

Eye symptoms in our cohort were high compared to other published studies. ${ }^{7-}$ 12 Ophthalmopathy is a common manifestation of Graves' disease and is present in up to $50 \%$ of patients. ${ }^{25}$ The effect of ophthalmopathy on quality of life has previously been reported using a variety of QOL questionnaires. ${ }^{26-28}$ Within our cohort, the incidence of Graves' disease was only $16.4 \%$. Given the small numbers in this study, subgroup analysis on Grave's patients was not performed, however post-operative eye symptom improvement has been reported in both studies of thyrotoxic patients ${ }^{12}$ and a number of mixed populations. ${ }^{7,8}$

It is interesting to note that a high proportion of patient's QOL was impaired in the mental health domains rather than physical domains. Baseline anxiety, depressivity, and cognition domain scores showed statistically significant improvement at six months postoperatively.-This is consistent with the published literature, which demonstrates significant post-operative improvement in anxiety scores both for toxic and non-toxic patients. ${ }^{3,8-12}$ Baseline questionnaires were administered on the day of pre-anaesthetic assessment and may reflect anxiety 
regarding upcoming surgery. The other mental domain, emotional susceptibility, did not improve post-operatively, contrary to the majority of published studies. ${ }^{8-12}$ While we recognize that having thyroid disease can be associated with symptoms related to those domains, it is not specific. One hypothesis for this improvement would be a placebo effect of having surgery, however it is not ethical to assess this with a trial. Many patients attribute any impairment in "mental domains" to their thyroid disease, so it is important to reinforce that surgery may not cure these symptoms during preoperative counselling.

Similar to Cramon et al and Bukvic et al, social impairment was the least affected domain post at baseline and six months post-operatively. ${ }^{7,8}$-There was no significant change in domain scores for impairment in social life and sex life. Results from published thyroidectomy ThyPRO studies ${ }^{7-10}$ show mixed results, however the majority reporting significant improvements in these domains. Interestingly, the cosmetic domain did not show significant post-operative change compared to baseline. Given a large proportion of our patients had surgery for a goitre, thyroidectomy would essentially swap a possibly visible lump for a scar.

Overall QOL improved following surgery and continued to improve at sixmonth follow up. It is reassuring that although all symptom domains didn't improve, , overall QOL was made better following surgery.

Analysis for demographic and clinical characteristics did not reveal any significant predictive factors for improved QOL. This suggests that improvements following thyroidectomy for benign disease can be expected regardless of 
characteristics such as age, gender and thyroid volume, and therefore is an acceptable treatment modality if the patient is fit for surgery. Certainly, patients who undergo total thyroidectomy for benign conditions will have no need for ongoing surveillance scans or follow up visits following the post-operative period.

\section{Strengths and Limitations}

There are a number of limitations to our study. Our study is small, and the attrition may present a source of bias. The analyses are based upon 72 patients with complete data. There were an additional 23 patients lost to follow up that did not complete all three questionnaires, who were slightly younger and more likely to be smokers than the study group. However, their baseline ThyPRO scores were similar, and they had less postoperative complications compared with study group. On that basis, we would not expect their outcomes to be worse than the study group if all questionnaires had been completed.

Postal surveys notoriously have low response rates, and patients who have had non-complicated thyroid surgery, may not require clinical follow up beyond 6 weeks, which may have impacted on the completeness of follow-up questionnaires. The original paper based ThyPRO questionnaire that we used is also quite cumbersome. A new, abbreviated version is now available which condenses the original 85 items to 39-items (ThyPRO-39), and the tool is now available electronically. ${ }^{30}$ Although all patients had benign thyroid disease, the pathology was 
heterogeneous. Due to the limited sample size it would be difficult to ascertain differences between groups across domains.

The strengths of this study include the prospective design and use of a disease-specific validated questionnaire. Despite its limitations, we believe our study provides contemporaneous information regarding the effect of benign thyroid surgery on QOL in Australia using a tool which may directly compared with other published studies.

In conclusion, our results show significant post-operative improvement in QOL following benign thyroid surgery using a validated, disease-specific questionnaire. Patients have a significant improvement in nine domains at six months after surgery: goitre symptoms, hyper- and hypothyroid symptoms, eye symptoms tiredness, anxiety, depressivity, cognition and daily life impairment. Importantly, surgery did not impair any QOL domain. With larger patient cohorts, we may be able to identify predictive factors for improvements in post-operative QOL for benign thyroid disease, allowing a more personalised assessment and risk profiling in the preoperative counselling of patients.

Author Disclosure Statement: No competing financial interests exist. 


\section{References}

1. Vanderpump MP 2011 The epidemiology of thyroid disease. Br Med Bull 99:39-51.

2. Grover G, Sadler G, Miahi R 2013Morbidity after thyroid surgery: patient perspective. Laryngoscope 123:2319-2323

3. Promberger R, Hermann M, Pallikunnel S, Seemann R, Meusel M, Ott J 2014 Quality of life after thyroid surgery in women with benign euthyroid goitre: influencing factors including Hashimoto's thyroiditis. Am J Surg 207:974-979.

4. Sabaretnam M, Mishra A, Chand G, Agarwal G, Agarwal A, Verma A, Mishra S 2012 Assessment of Swallowing Function Impairments in Patients with Benign Goitres and Impact of Thyroidectomy: a case control study. World J Surg 36:1293-

5. Scerrino G, Mortino G, Paladion N, Di Paola V, Amodio E, Gulotta G, Bonventre S 2013 Does thyroid surgery for Graves' disease improve healthrelated quality of life? Surg. Today 43:1398-1405.

6. Greenblatt D, Sippel R, Leverson G, Frydman J, Schaefer S, Chen H 2009 Thyroid resection improves perceptions of swallowing function in patients with thyroid disease. World J Surg 33:255-26

7. Cramon P, Bonnema SJ, Bjorner JB, Ekholm O, Feldt-Rasmussen U, Frendl M, Groenvold M, Hegedüs L, Rasmussen K, Watt T 2015 Quality of life in 
patients with benign nontoxic goitre: impact of disease and treatment response, and comparison with the general population. Thyroid 25(3):284-291

8. Bukvic B, Zivaljevic V, Sipetic S, Diklic A, Tausanovic K, Paunocic I 2014 Improvement of quality of life in patients with benign goitre after surgical treatment. Lagenbecks Arch Surg 299:755-765

9. Mishra A, Sabaretnam M, Chand G, Agarwal G, Agarwal A, Verma A, Mishra S 2013 Quality of life in Patients with Benign Thyroid Goitres (Pre- and PostThyroidectomy): A Prospective Study. World J Surg 37:2322-2329

10. Sorensen J, Watt T, Cramon P, Døssing H, Hegedüs L, Bonnema S, Godballe C 2017 Quality of life after thyroidectomy in patients with nontoxic nodular goitre: a prospective cohort study. Head and Neck 39:2232-2240

11. Zivaljevic V, Bacotic B, Sipetic S, Stanisavljevic D, Maksimovic J, Diklic A, Paunovic I 2015 Quality of life improvement in patients with Hashimoto thyroiditis and other goitres after surgery: A prospective cohort study. Int J Surg 21:150-155

12. Bukvic B, Zivaljevic V, Sipetic S, Diklic A, Tausanovic K, Stojanovic D, Stevanovic D, Paunovic I 2015 Improved quality of life in hyperthyroidism patients after surgery. Journal of Surgical Research 193:724-730

13. Watt T, Bjorner JB, Groenvold M, Rasmussen AK, Bonnema SJ, Hegedus L,Feldt-Rasmussen U 2009 Establishing construct validity for the thyroidspecific patient reported outcome measure (ThyPRO): an initial examination. Qual Life Res 18(4):483-496 
14. Watt T, Hegedüs L, Rasmussen AK, Groenvold M, Bonnema SJ, Bjorner JB, Feldt-Rasmussen U 2007 Which domains of thyroid-related quality of life are most relevant? Patients and clinicians provide complementary perspectives. Thyroid 17(7):647-654

15. Watt T, Hegedus L, Groenvold M, Bjorner JB, Rasmussen AK, Bonnema SJ, Feldt-Rasmussen U 2010 Validity and reliability of the novel thyroid-specific quality of life questionnaire, ThyPRO. Eur J Endocrinol 162:161-167

16. Watt T, Cramon P, Hegedus L, Bjorner JB, Bonnema SJ, Rasmussen AK, Feldt-Rasmussen U, Groenvold M 2014 The thyroid-related quality of life measure ThyPRO has good responsiveness and ability to detect relevant treatment effects. J Clin Endocrinol Metab 99:3708-3717

17. Watt T, Groenvold M, Deng N, Gandek B, Feldt-Rasmussen U, Rasmussen AK, Hedegus L, Bonnema SJ, Bjorner JB 2014 Confirmatory factor analysis of the thyroid-related quality of life questionnaire ThyPRO. Health Qual Life Outcomes 12:126

18. Watt T, Groenvold M, Hegedus L, Bonnema SJ, Rasmussen AK, FeldtRasmussen U, Bjorner JB 2014 Few items in the thyroid-related quality of life instrument ThyPRO exhibited differential item functioning. Qual Life Res 23:327-338

19. Wong C, Lang B, Lam C 2016 A systematic review of quality of thyroidspecific health-related quality-of-life instruments recommends ThyPRO for patients with benign thyroid diseases. J Clin Epidemiol 78: 63-72 
20. R Core Team (2019). R: A language and environment for statistical computing. R Foundation for Statistical Computing, Vienna, Austria. URL https://www.R-project.org/.

21. Pinheiro J, Bates D, DebRoy S, Sarkar D, R Core Team (2019). nlme: Linear and Nonlinear Mixed Effects Models. R package version 3.1-142, URL https://CRAN.R-project.org/package=nlme.

22. Dripps RD 1963 New classification of physical status. Anesthesiol 24:111

23. Pernambuco L, Almeida M, Matias K, Costa E 2015 Voice Assessment and Voice-related Quality of life in Patients with Benign Thyroid Disease. Otolarynol Head Neck Surg 152(1):116-121

24. Gohrbandt A, Aschoff A, Gohrbandt B, Keilmman A, Lang H, Musholt T 2016 Changes of Laryngeal Mobility and Symptoms Following Thyroid surgery: 6month Follow-up. World J Surg 40:636-643

25. Wiersinga W, Bartalene L 2002 Epidemiology and prevention of Graves' ophthalmopathy. Thyroid 12:855-860

26. Farid M, Roch-Levecq A, Levi L, Brody B, Granet D, Kikkawa D 2005 Psychological disturbance in graves' ophthalmopathy. Arch Ophthalmol 123(4):491-6.

27. Terwee C, Wakelkamp I, Tan S, Dekker F, Prummel M, Wiersinga W 2002 Long term effects of Graves' ophthalmopathy on health-related quality of life. Eur J Endocrinol 146(6):751-7

This article is protected by copyright. All rights reserved. 
28. Coulter I, Frewin S, Krassas G, Perro P 2007 Psychological implications of Graves' orbitopathy. Eur J Endocrinol 157(2):127-31

29. Watt T, Bjorner JB, Groenvold M, Cramon P, Winther $\mathrm{KH}$, Hegedüs L, Bonnema SJ, Rasmussen ÅK, Ware JE Jr, Feldt-Rasmussen U 2015 Development of a Short Version of the Thyroid-Related Patient-Reported Outcome ThyPRO. Thyroid 25(10):1069-79

30. Rasmussen S, L, Rejnmark L, Ebbehøj E, Feldt-Rasmussen U, Rasmussen Å, K, Bjorner J, B, Watt T 2016 High Level of Agreement between Electronic and Paper Mode of Administration of a Thyroid-Specific Patient-Reported Outcome, ThyPRO. Eur Thyroid J 5:65-72 
Table 1. Patient Demographics

Table 2. Sample Clinical Characteristics

Table 3. Modelled estimates of Change in ThyPRO Scores over Time

Table S1. Comparison of demographics and clinical characteristics of analysis sample and lost to follow up sample

Table S2. Comparison of baseline ThyPRO scores, analysis sample and lost to follow up sample

Table S3. Sub-scale Scores for ThyPRO 
Table 3. Modelled estimates of Change in ThyPRO Scores over Time

\section{Scale}

Goitre Symptoms

Hyperthyroid Symptoms Hypothyroid Symptoms

Eye Symptoms

Tiredness

Cognitive Problems Anxiety

Depressivity

Emotional Susceptibility

Social Impairment

Impaired Daily Life

Impaired Sex Life

Cosmetic Complaints

\begin{tabular}{|c|c} 
& bas \\
& Estimate $^{\mathbf{a}}$ \\
-11.3 \\
-12.1 \\
-7.0 \\
-5.4 \\
-7.8 \\
-3.8 \\
-10.6 \\
-3.0 \\
-3.9 \\
1.0 \\
-6.5 \\
0.7 \\
-3.0 \\
\end{tabular}

baseline to 6 weeks

$\begin{array}{cc}\text { 95\% Cl } & \text { p value } \\ -17.4,-5.2 & <0.005^{\star} \\ -16.6,-7.6 & <0.005^{\star} \\ -11.8,-2.1 & <0.005^{\star} \\ -9.4,-1.3 & <0.01^{*} \\ -13.9,-1.8 & 0.01^{*} \\ -8.1,0.6 & 0.09 \\ -15.7,-5.4 & <0.005^{\star} \\ -7.9,1.9 & 0.231 \\ -8.6,0.8 & 0.104 \\ -3.8,5.7 & 0.692 \\ -12.1,-0.9 & 0.022^{\star} \\ -6.5,7.9 & 0.849 \\ -9.1,3.1 & 0.333\end{array}$

$-9.1,3.1 \quad 0.333$
6 weeks to 6 months

$\begin{array}{cc}\text { Estimate }^{b} & 95 \% \mathbf{C l} \\ -10.7 & -14.3,-7.1 \\ 1.2 & -1.8,4.2 \\ 1.2 & -2.8,5.1 \\ -1.2 & -4.4,2.0 \\ 1.4 & -4.5,7.3 \\ -3.1 & -7.3,1.0 \\ 0.3 & -3.6,4.2 \\ -1.9 & -5.9,2.2 \\ -1.1 & -5.0,2.8 \\ -3.5 & -8.2,1.2 \\ -0.7 & -4.6,3.3 \\ 1.7 & -5.5,8.8 \\ -3.0 & -7.9,1.8\end{array}$

baseline to 6 months

$\begin{array}{ccc}\text { Estimate }^{\mathrm{c}} & 95 \% \mathrm{Cl} & \mathbf{p ~ v a l u e} \\ -22.0 & -27.3,-16.8 & <0.005^{*} \\ -10.9 & -15.3,-6.5 & <0.005^{\star} \\ -5.8 & -10.2,-1.3 & 0.01^{*} \\ -6.5 & -10.3,-2.8 & <0.005^{*} \\ -6.5 & -12.3,-0.7 & 0.03^{*} \\ -6.9 & -11.7,-2.1 & <0.005^{\star} \\ -10.3 & -15.6,-5.0 & <0.005^{\star} \\ -4.9 & -9.2,-0.5 & 0.02^{*} \\ -5.0 & -10.1,0.04 & 0.052 \\ -2.6 & -7.6,2.5 & 0.32 \\ -7.2 & -12.4,-1.9 & <0.01^{*} \\ 2.4 & -6.2,10.9 & 0.59 \\ -6.0 & -12.8,0.7 & 0.07\end{array}$

* indicates $p<0.05$

a. estimate is the difference in modelled scores ( 6 weeks minus baseline)

b. estimate is the difference in modelled scores ( 6 months minus 6 weeks)

c. estimate is the difference in modelled scores ( 6 months minus baseline)

$\mathrm{Cl}=$ confidence interval 
Table 1. Patient Demographics

\begin{tabular}{lc}
\hline & $\mathbf{N}=\mathbf{7 2}$ \\
\hline Age in years, mean (SD) & $63(87.5)$ \\
Female gender, $\mathbf{n}(\%)$ & $35(48.6)$ \\
Country of Birth & $37(51.4)$ \\
Australia $\mathbf{n}(\%)$ & \\
Other $\mathbf{n}(\%)$ & $17(23.6)$ \\
& \\
Current smoker, $\mathbf{n}(\%)$ & $15(20.8)$ \\
BMl & $28(38.9)$ \\
Healthy weight (18 - 24.9), $\mathbf{n}(\%)$ & $29(40.3)$ \\
Overweight (25 - 29.9) $\mathbf{n}(\%)$ & \\
Obese (>30) $\mathbf{n}(\%)$ & \\
\hline
\end{tabular}

This article is protected by copyright. All rights reserved. 
Table 2. Sample Clinical Characteristics

$\mathrm{N}=72$

\begin{tabular}{lc}
\hline Pre-operative Thyroid Function, $\mathbf{n}(\%)$ & $20(27.8)$ \\
Hyperthyroid & $51(70.8)$ \\
Euthyroid & $1(1.4)$ \\
Hypothyroid & $36(50.0)$ \\
Indication for Surgery, $\mathbf{n}(\%)$ & $12(16.7)$ \\
Multi-nodular Goitre & $6(8.3)$ \\
Graves' Disease & $18(25.0)$ \\
Toxic Nodule/Goitre & \\
Nodule for Investigation & $15(21.7)$ \\
ASA Score†(23) & $40(58.0)$ \\
ASA 1 & $14(20.3)$ \\
ASA 2 & \\
ASA 3 & $43(59.7)$ \\
Operation Performed & $2(2.8)$ \\
Hemi-thyroidectomy & $27(37.5)$ \\
Completion Thyroidectomy & \\
Total Thyroidectomy & $52(72.2)$ \\
Histopathology & $10(13.9)$ \\
Multinodular goitre & $2(2.8)$ \\
Grave's Disease & $1(1.4)$ \\
Thyroiditis & $3(4.2)$ \\
Cyst & $4(5.6)$ \\
Hyperplastic Nodule & \\
Solitary Adenoma & $1(1.4)$ \\
Surgical Complications & $0(0)$ \\
Temporary RLN palsy & $18(25)$ \\
Permanent RLN Palsy & $1(1.4)$ \\
Temporary hypoparathyroidism & \\
Permanent hypoparathyroidism & \\
& \\
† Only available for 69 patients & \\
\end{tabular}

This article is protected by copyright. All rights reserved. 


\section{University Library}

\section{- M M N E R VA A gateway to Melbourne's research publications}

Minerva Access is the Institutional Repository of The University of Melbourne

Author/s:

Chew, CR;Chin, SL;Lam, T;Drosdowsky, A;Chan, STF;Chin-Lenn, L

Title:

How does thyroidectomy for benign thyroid disease impact upon quality of life? A prospective study

Date:

2020-09-25

\section{Citation:}

Chew, C. R., Chin, S. L., Lam, T., Drosdowsky, A., Chan, S. T. F. \& Chin-Lenn, L. (2020). How does thyroidectomy for benign thyroid disease impact upon quality of life? A prospective study. ANZ JOURNAL OF SURGERY, 90 (12), pp.E177-E182. https://doi.org/10.1111/ ans. 16342.

Persistent Link:

http://hdl.handle.net/11343/276353 